\title{
A multiscale phenomenological constitutive model for strain rate dependent tensile ductility in nanocrystalline metals
}

\author{
M. Amir Siddiqa, ${ }^{\mathrm{a}}$, Tamer El Sayed ${ }^{\mathrm{b}}$ \\ ${ }^{a}$ School of Engineering, University of Aberdeen, Fraser Noble Building, AB24 3UE, Aberdeen, United \\ Kingdom \\ ${ }^{b}$ Officio, Budapest, Hungary \\ *Corresponding Author:muhammad.amir@abdn.ac.uk
}

\begin{abstract}
A variational multiscale constitutive model that accounts for strain rate dependent ductility of nanocrystalline materials during intergranular failure has been presented. The presented model is an extension of the previous work [1], in which a nanocrystalline material is modelled as two-phase with grain interior being modelled using crystal plasticity theory while grain boundary affected zone using porous plasticity model which accounts for ductile damage due to void growth and coalescence. The model capability of capturing the strain rate dependent deformation and failure has been demonstrated through validations against uniaxial test data taken from literature. The validated results show a good agreement between experimental and simulated response.
\end{abstract}

Keywords: Variational Updates, Nanocrystalline materials, crystal plasticity theory, Intergranular failure, strain rate sensitivity

\section{Introduction}

Enhanced strain rate sensitivity of yield stress and ductility has been observed in nanocrystalline materials in the recent past [2]-[16]. It has been reported that high diffusivity in grain boundaries enhances void growth and coalescence [17], [18]. It has also been reported that in nanocrystalline metals changing the strain rate changes the ductility [2]-[7]. Numerical simulations of deformation and rate independent failure in nanocrystalline materials have been performed in the recent past [19]-[23]. Siddiq and El Sayed [1] presented rate independent constitutive model for intergranular failure in nanocrystalline materials by considering void growth and coalescence in the grain boundary region.

In the present work, the rate independent model proposed in Siddiq and El Sayed [1] is extended to rate dependent case. The model comprises of a grain boundary affected zone and grain interior. Similar to Siddiq and El Sayed [1], grain boundary affected zone is modeled using the fully variational rate sensitive porous plasticity model with void growth and coalescence during failure.

\section{Constitutive Model Framework}

The classical model proposed by Gurses and El Sayed [23], [24], [26] and Siddiq and El Sayed [1] comprises of two phases for each grain. Each grain consists of a single crystalline core where deviatoric 
dislocation mediated plasticity is the dominating deformation mechanism and grain boundary affected zone where atomic shuffling, grain boundary sliding, void growth and coalescence are operative [1].

The extended model is briefly discussed in the following with major modifications. Details about the variational formulation of the model can be found in Siddiq and El Sayed [1].

Plastic flow rule is defined as

$$
\dot{F}_{g b}^{p} \dot{F}_{g b}^{p-1}=\dot{\varepsilon}^{p} \mathrm{M}+\dot{\theta}^{p} \mathrm{~N}+\beta \dot{\eta}^{p} N
$$

Where $\varepsilon^{p}$ is the deviatoric plastic strain, $\theta^{p}$ is the volumetric plastic strain, $\eta^{p}$ accounts for the void coalescence and is the percentage of voids undergoing coalescence at a given material point, $\beta$ is a material constant that transforms $\eta^{p}$ into a variable that measures volumetric strain, $\mathbf{M}$ and $\mathbf{N}$ are directions of the deviatoric and volumetric plastic deformation rates, respectively.

Based on the experimental findings [6], [10], [27], it is assumed that plastic part of the deformation and the damage initiation at the onset of coalescence are strongly dependent upon the strain rates while damage evolution after the onset of void coalescence is rate independent. Rate-dependent plasticity is incorporated using a rate potential [23], [24], [26] while after the onset of the coalescence rate independent potentials have been used [1]. Rate dependent potentials, before the onset of coalescence, are given by

$$
\begin{gathered}
\Psi_{g b}^{*}\left(\dot{\varepsilon}^{p}, \dot{\theta}^{p}, J^{p}\right)=\Psi_{g b}^{*, v o l}\left(\dot{\theta}^{p}, J^{p}\right)+\Psi_{g b}^{*, d e v}\left(\dot{\varepsilon}^{p}\right) \\
\Psi_{g b}^{*, v o l}\left(\dot{\theta}^{p}, J^{p}\right)=\frac{m^{2} \sigma_{0} \dot{\varepsilon}_{0}^{p}}{m+1} N \frac{4 \pi a^{3}}{3}\left(1-f^{1 / m}\right)\left|\frac{2 \dot{a}}{\dot{\varepsilon}_{0}^{p} a}\right|^{\frac{m+1}{m}} \\
\Psi_{g b}^{*, d e v}\left(\dot{\varepsilon}^{p}\right)=\frac{m \sigma_{0} \dot{\varepsilon}_{0}^{p}}{m+1}\left(\frac{\dot{\varepsilon}^{p}}{\dot{\varepsilon}_{0}^{p}}\right)^{\frac{m+1}{m}}
\end{gathered}
$$

Where $m$ is the rate sensitivity exponent, $\dot{\varepsilon}_{0}^{p}$ is the reference plastic strain rate, $N$ is the void density, $f$ is the volume fraction of voids, $a$ is the mean void radius and $\sigma_{0}$ is the yield stress.

For the criteria to onset void coalescence in the grain boundary region, strain rate dependent critical porosity is given by

$$
f_{c}=A \dot{\varepsilon}^{B}
$$

Where $A$ and $B$ are material parameters identified from experimental stress-strain data.

After the onset of the void coalescence, the model assumes that grain boundary zone becomes rate independent and uses rate independent energy potentials given by 


$$
\begin{gathered}
W_{\text {growth }, g b}^{p, v o l}\left(\theta^{p}, \eta^{p}\right)=\frac{n \sigma_{0} \varepsilon_{0}^{p}}{n+1}\left(1-\eta^{p}\right)\left(N_{0} \frac{4 \pi a^{3}}{3}\right) \bar{g}\left(\theta^{p}, \eta^{p}\right) \\
W_{\text {coal,gb }}^{p, v o l}\left(\theta^{p}, \eta^{p}\right)=\frac{n \sigma_{0} \varepsilon_{0}^{p}}{n+1}\left(\eta^{p}\right)\left(N_{0} \frac{4 \pi \tilde{a}^{3}}{3}\right) \tilde{g}\left(\theta^{p}, \eta^{p}\right) \\
\bar{g}=\int_{1}^{1 / f}\left(1+\frac{2}{3 \varepsilon_{0}^{p}} \log \frac{x}{x-1+\frac{\gamma f_{0}}{f_{0}+e^{\left(\theta^{p}+\beta \eta^{p}\right)}-1}}\right)^{\frac{n+1}{n}} d x \\
\tilde{g}=\int_{1}^{1 / f_{a}}\left(1+\frac{2}{3 \varepsilon_{0}^{p}} \log \frac{x}{x-1+\frac{\gamma f_{0} /(\tilde{a} / a)^{3}}{f_{0}+e^{\left(\theta p+\beta \eta^{p}\right)}-1}}\right)^{\frac{n+1}{n}} d x
\end{gathered}
$$

Where $n$ is the hardening exponent. $\tilde{a} / a$ and $\curlyvee$ are given by

$$
\begin{aligned}
& \left(\frac{\tilde{a}}{a}\right)^{3}=1+\alpha_{1}\left(f-f_{c}\right)^{\alpha_{2}} \\
& \gamma=\left(1-\eta^{p}\right)+\eta^{p}\left(\frac{\tilde{a}}{a}\right)^{3}
\end{aligned}
$$

Where $\alpha_{1}$ and $\alpha_{2}$ are materials parameters.

It must be noted that strain rate dependent critical porosity along with the void coalescence parameters, discussed above, will control the strain rate dependent ductility of the nanocrystalline materials during intergranular failure. For detailed discussion about the above constitutive model, please see [1], [23], [24], [26] and references therein.

\section{Results and Discussions}

The applicability of the presented constitutive model is shown by performing simulations on nanocrystalline materials. Brief description of individual experiments and validated results are presented in the following.

Dalla Torre et al. [6] performed studies on electroplated nanocrystalline nickel ( $\mathrm{Ni}$ ) foils at strain rates between $10^{-5}$ and $10^{3} \mathrm{~s}^{-1}$. Comparison between experimental and simulated response is shown in Figure 1 while identified set of parameters are shown in Table 1. Results show a good agreement between experimental and simulated response. 


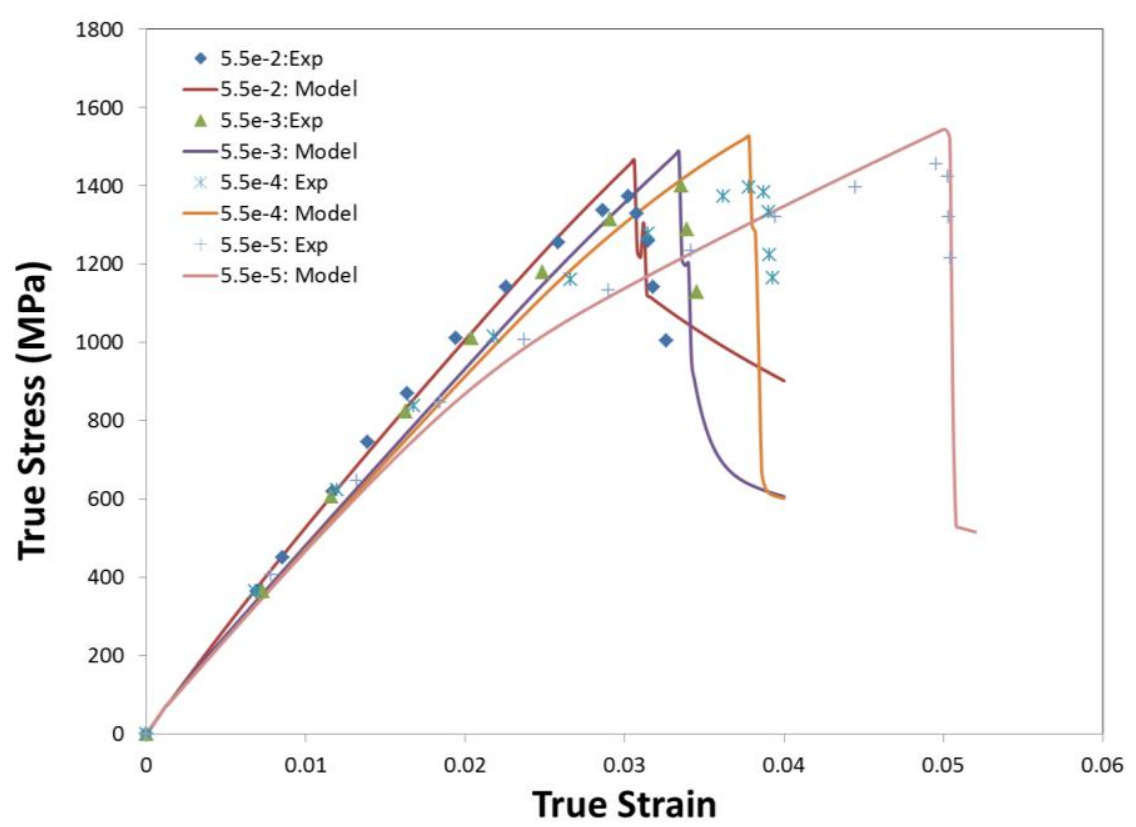

Figure 1: Comparison of model prediction and experimental data reported in Dalla Torre et al. [6]

Table 1: Material parameters identified using inverse modelling approach

\begin{tabular}{llll}
\hline Material Properties & Dalla Torre et al. [6] & Zhang et al. [10] & Schwaiger et al. [27] \\
\hline $\mathrm{E}(\mathrm{GPa})$ & $92.73 \mathrm{~d} 3$ & $85 . \mathrm{d} 3$ & $182.7 \mathrm{~d} 3$ \\
$\boldsymbol{\nu}$ & $0.31 \mathrm{~d} 0$ & $0.34 \mathrm{~d} 0$ & $0.31 \mathrm{~d} 0$ \\
$\boldsymbol{\sigma}_{\mathbf{0}}\left(\boldsymbol{T}_{0}\right)$ & 40 & 43 & 450 \\
$\boldsymbol{\varepsilon}_{\mathbf{0}}^{\boldsymbol{p}}$ & $0.0113 \mathrm{~d} 0$ & $0.01 \mathrm{~d} 0$ & $0.013 \mathrm{~d} 0$ \\
$\dot{\boldsymbol{p}}$ & 0.002 & 0.02 & 0.012 \\
$\boldsymbol{\varepsilon}_{\mathbf{0}}$ & $1 . \mathrm{d} 0$ & $5.1 \mathrm{~d} 0$ & $25.0 \mathrm{~d} 0$ \\
$\boldsymbol{m}$ & $1.75 \mathrm{~d} 0$ & $2.3 \mathrm{~d} 0$ & $4.5 \mathrm{~d} 0$ \\
$\boldsymbol{n}$ & $5.00 \mathrm{E}-07$ & $7.60 \mathrm{E}-07$ & $1.00 \mathrm{E}-06$ \\
$\boldsymbol{a}_{0}$ & $1.00 \mathrm{E}+15$ & $1.00 \mathrm{E}+16$ & $1.00 \mathrm{E}+16$ \\
$\boldsymbol{N}_{\boldsymbol{0}}$ & 0.0001 & 0.01 & 0.1 \\
$\boldsymbol{\alpha}_{\mathbf{1}}$ & 10 & 10.8 & 0.1 \\
$\boldsymbol{\alpha}_{\mathbf{2}}$ & 0.05 & 0.05 & 0.012 \\
$\boldsymbol{\beta}$ & 0.58 & 0.01 & 0.16 \\
$\mathbf{A}$ & 3 & 3 & 3 \\
\hline B & & & \\
\hline
\end{tabular}

Schwaiger et al. [27] performed tensile testing of ultrafine grain Ni between strain rates $3 e-4$ to $3 e-1 \mathrm{~s}^{-1}$. It was reported that ultra-fine grain $\mathrm{Ni}$ showed significant rate sensitivity over the selected strain rate range. It was also suggested that this could be because of rate-sensitive grain-boundary affected zone. The results of the comparison of experimental and simulated stress-strain response are plotted in Figure 2 while parameters identified are shown in Table 1. 


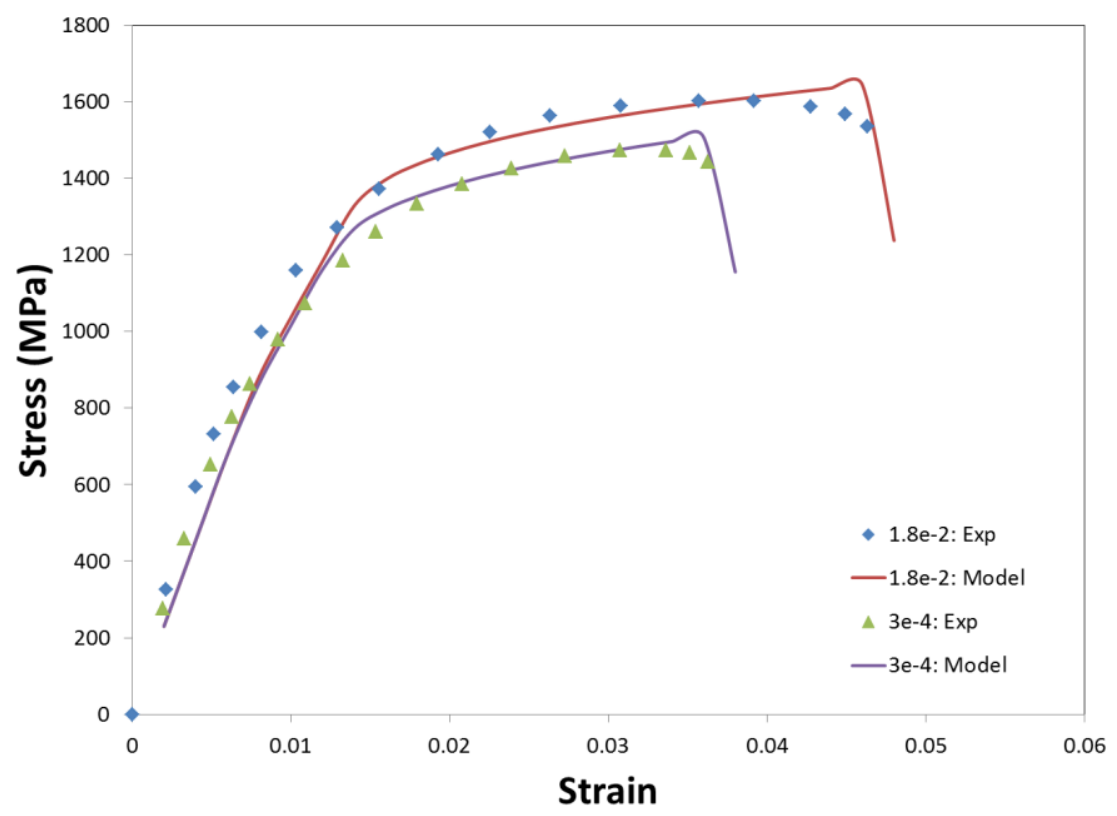

Figure 2: Comparison of model prediction and experimental data reported in Schwaiger et al. [27]

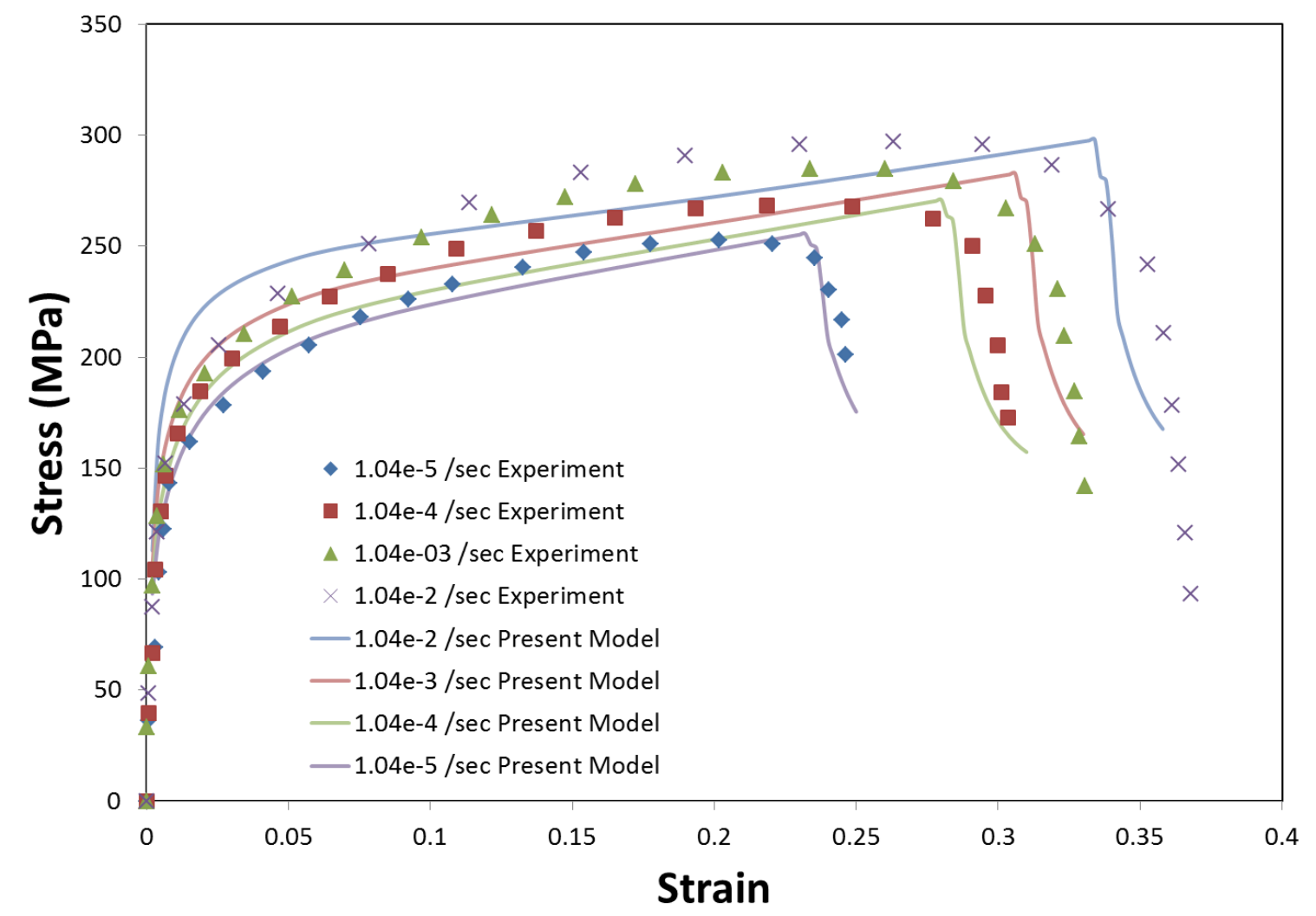

Figure 3: Comparison of model prediction and experimental data reported in Zhang et al. [10]

Zhang et al. [10] performed experimental studies to understand tensile behavior of ultrafine grain Copper (Cu). It was reported that as the strain rate is increased ductility is also increased. A comparison 
between model predictions and experimental results are presented in Figure 3 shows a decent agreement.

\section{Conclusions}

A fully variational multiscale constitutive model for strain rate dependent ductility of nanocrystalline metals was presented. Numerical validations were presented based on inverse modelling approach and showed a good agreement with experiments. As a future work, experimentalists have reported strain rate dependent grain growth in the literature and this model is planned to be extended to account for strain rated dependent grain growth in nanocrystalline metals.

\section{Acknowledgements}

No external funding was received for this project.

\section{References}

[1] A. Siddiq and T. El Sayed, "A phenomenological variational multiscale constitutive model for intergranular failure in nanocrystalline materials," Mater. Lett., vol. 107, pp. 56-59, Sep. 2013.

[2] J. Chen, L. Lu, and K. Lu, "Hardness and strain rate sensitivity of nanocrystalline Cu," Scr. Mater., vol. 54, no. 11, pp. 1913-1918, Jun. 2006.

[3] S. CHENG, E. MA, Y. WANG, L. KECSKES, K. YOUSSEF, C. KOCH, U. TROCIEWITZ, and K. HAN, "Tensile properties of in situ consolidated nanocrystalline Cu," Acta Mater., vol. 53, no. 5, pp. 1521-1533, Mar. 2005.

[4] Z. Jiang, X. Liu, G. Li, Q. Jiang, and J. Lian, "Strain rate sensitivity of a nanocrystalline Cu synthesized by electric brush plating," Appl. Phys. Lett., vol. 88, no. 14, p. 143115, Apr. 2006.

[5] R. K. Guduru, K. L. Murty, K. M. Youssef, R. O. Scattergood, and C. C. Koch, "Mechanical behavior of nanocrystalline copper," Mater. Sci. Eng. A, vol. 463, no. 1-2, pp. 14-21, Aug. 2007.

[6] F. D. Torre, H. V Swygenhoven, and M. Victoria, "Nanocrystalline electrode-posited Ni: Microstructure and tensile properties.," Acta Mater., vol. 50, pp. 3957-3970, 2002.

[7] C. Gu, J. Lian, Z. Jiang, and Q. Jiang, "Enhanced tensile ductility in an electrodeposited nanocrystalline Ni," Scr. Mater., vol. 54, no. 4, pp. 579-584, Feb. 2006.

[8] L. Lu, S. X. Li, and K. Lu, "An abnormal strain rate effect on tensile behavior in nanocrystalline copper," Scr. Mater., vol. 45, no. 10, pp. 1163-1169, Nov. 2001.

[9] V. M. Segal, "Deformation mode and plastic flow in ultra fine grained metals," Mater. Sci. Eng. A, vol. 406, no. 1-2, pp. 205-216, Oct. 2005. 
[10] H. Zhang, Z. Jiang, J. Lian, and Q. Jiang, "Strain rate dependence of tensile ductility in an electrodeposited Cu with ultrafine grain size," Mater. Sci. Eng. A, vol. 479, no. 1-2, pp. 136-141, Apr. 2008.

[11] Y. Wei, C. Su, and L. ANAND, "A computational study of the mechanical behavior of nanocrystalline fcc metals.," Acta Mater., vol. 54, no. 12, pp. 3177-3190, Jul. 2006.

[12] S. Van Petegem, J. Zimmermann, and H. Van Swygenhoven, "Yield point phenomenon during strain rate change in nanocrystalline Ni-Fe," Scr. Mater., vol. 65, no. 3, pp. 217-220, Aug. 2011.

[13] M. A. E. Meyers, A. Mishra, and D. J. J. Benson, "Mechanical properties of nanocrystalline materials," Prog. Mater. Sci., vol. 51, no. 4, pp. 427-556, May 2006.

[14] H. Li, J. Zhou, R. Zhu, and X. Ling, "The evolution of porosity in bulk nanocrystalline materials during plastic deformation and its effect on the mechanical behavior," Mater. Des., vol. 31, no. 2, pp. 1003-1006, Feb. 2010.

[15] K. Jonnalagadda, N. Karanjgaokar, I. Chasiotis, J. Chee, and D. Peroulis, "Strain rate sensitivity of nanocrystalline Au films at room temperature," Acta Mater., vol. 58, no. 14, pp. 4674-4684, Aug. 2010.

[16] P. Huang, F. Wang, M. Xu, K. W. Xu, and T. J. Lu, "Dependence of strain rate sensitivity upon deformed microstructures in nanocrystalline Cu," Acta Mater., vol. 58, no. 15, pp. 5196-5205, Sep. 2010.

[17] K. S. S. Kumar, S. Suresh, M. F. F. Chisholm, J. A. A. Horton, and P. Wang, "Deformation of electrodeposited nanocrystalline nickel.," Acta Mater., vol. 51, no. 2, pp. 387-405, Jan. 2003.

[18] K. S. Kumar, H. Van Swygenhoven, and S. Suresh, "Mechanical behavior of nanocrystalline metals and alloys.," Acta Mater., vol. 51, pp. 5743-5774, 2003.

[19] I. V. Alexandrov, R. G. Chembarisova, and V. D. Sitdikov, "Analysis of the deformation mechanisms in bulk ultrafine grained metallic materials," Mater. Sci. Eng. A, vol. 463, no. 1-2, pp. 27-35, Aug. 2007.

[20] H.-H. Fu, D. J. Benson, and M. André Meyers, "Computational description of nanocrystalline deformation based on crystal plasticity," Acta Mater., vol. 52, no. 15, pp. 4413-4425, Sep. 2004.

[21] Y. WEI, C. SU, and L. ANAND, "A computational study of the mechanical behavior of nanocrystalline fcc metals," Acta Mater., vol. 54, no. 12, pp. 3177-3190, Jul. 2006.

[22] C. Schillebeeckx, S. Berbenni, L. Capolungo, and M. Cherkaoui, "A new micromechanics-based scale transition model for the strain-rate sensitive behavior of nanocrystalline materials," Philos. Mag., vol. 91, no. 5, pp. 657-681, Feb. 2011.

[23] E. Gürses and T. El Sayed, "Constitutive modeling of strain rate effects in nanocrystalline and ultrafine grained polycrystals," Int. J. Solids Struct., vol. 48, no. 10, pp. 1610-1616, May 2011. 
[24] E. Gurses, T. El Sayed, and E. Gürses, "A variational multiscale constitutive model for nanocrystalline materials.," J. Mech. Phys. Solids, vol. 59, no. 3, pp. 732-749, Mar. 2011.

[25] T. Gurses E. El Sayed, "A constitutive model of nanocrystalline metals based on competing grain boundary and grain interior deformation mechanisms," Mater. Lett., vol. 65, pp. 3391-3395, 2011.

[26] E. Gürses and T. El Sayed, "A constitutive model of nanocrystalline metals based on competing grain boundary and grain interior deformation mechanisms," Mater. Lett., vol. 65, no. 23-24, pp. 3391-3395, Dec. 2011.

[27] R. Schwaiger, B. Moser, M. Dao, N. Chollacoop, and S. Suresh, "Some critical experiments on the strain-rate sensitivity of nanocrystalline nickel.," Acta Mater., vol. 51, no. 17, pp. 5159-5172, Oct. 2003. 